\title{
Twenty years of RNA and mRNA decay
}

\section{MEGERDITCH KILEDJIAN}

Department of Cell Biology and Neuroscience, Rutgers University, Piscataway, New Jersey 08854-8082, USA

Happy 20th anniversary RNA journal! It is hard to believe it has already been two decades since the inception of the journal. I still remember the excitement within the RNA community upon hearing that the RNA Society would have its own journal. Over the years, RNA has proven itself as a major venue for disseminating quality research on RNA biology and a noteworthy contributor to the recognition of the significance of RNA research and the RNA field in general. Progress in the past 20 years has been remarkable in establishing the significance of post-transcriptional control and has fueled the realization that regulated gene expression does not end following synthesis of a pre-mRNA. For example, two decades ago, microRNAs were still in their infancy and more generally thought of as an anomaly while RNA interference and the plethora of subsequently described noncoding RNAs, had not yet been reported and likely had not yet even been envisioned. The realization that much of these transcripts could be regulatory RNAs has redefined the RNA landscape. The tremendous potential unleashed by these endogenous or exogenous small RNAs has opened a whole new frontier to enable functional dissection of gene expression not previously possible in mammalian cells. Moreover, uncovering the amazing ability of prokaryotes to use an elaborate RNA-based innate immune response encoded within the Clustered Regularly Interspaced Short Palindromic Repeats (CRISPR) has arguably provided one of the most revolutionary means to alter and assess eukaryotic gene expression. One underlying principle common to all RNAs is that they are not around forever. They all have distinct half-lives and are ultimately degraded. The fact that the overwhelming majority of our genome is transcribed into RNAs of unknown functions, all of which must ultimately be degraded, illustrates the significant role that RNA turnover plays in normal cellular homeostasis. Much of our current knowledge of RNA turnover is focused on the degradation of mRNAs. A brief flashback to the mid-'90s, current status and future direction of mRNA decay research follows below.

\section{Where we were in mRNA decay}

Much of what we knew of how mRNA degradation occurred in 1995 was primarily from studies in yeast. It was clear that mRNAs degraded through ordered pathways and were not randomly destroyed and the protective structures on the termini of mRNAs, the $5^{\prime} \mathrm{m} 7 \mathrm{G}$ cap and $3^{\prime}$ poly(A) tail needed to first be removed. An mRNA was known to undergo removal of the poly(A) tail (deadenylation) as the initial step followed by decay from one of the termini. From the $5^{\prime}$ end, the deadenylated mRNA would be decapped to remove the protective cap and expose the $5^{\prime}$ end to exonucleolytic decay by Xrn 1 . In the 3'-end decay pathway, the mRNA body would be degraded by an unknown exonucleolytic activity. Although XrnI was known to play a role in RNA decay, the remaining nucleases and axillary factors were not identified. In mammalian cells, deadenylation was known to be an initial step, but the subsequent steps had not yet been determined although were speculated to be similar.

Our knowledge of regulated mRNA decay was primarily restricted to observations that transcripts harboring an AUrich element (ARE) in their $3^{\prime}$ UTR could promote rapid mRNA decay and transcripts with a premature translation termination codon were also unstable and targeted by the nonsense-mediated mRNA decay (NMD) pathway. The ARE, initially detected in labile cytokine and proto-oncogene mRNAs, had been shown to promote deadenylation and promote rapid decay of these mRNAs. However, the identity of the factors and the mechanism involved were unknown. In the NMD quality control pathway, the involvement of several genetically identified genes had been established. These predominantly consisted of the Up Frameshift (UPF) proteins and the Suppressor with Morphogenetic effect on Genitalia (SMG) proteins. The molecular mechanism by which they imparted detection and decay of premature codon containing mRNAs was still elusive. Albeit an oversimplification, the above description sums up the state of the field circa 1995.
Corresponding author: kiledjian@biology.rutgers.edu

Article and publication date are at http://www.rnajournal.org/cgi/doi/10. 1261/rna.050112.115. Freely available online through the RNA Open Access option.
(C) 2015 Kiledjian This article, published in $R N A$, is available under a Creative Commons License (Attribution-NonCommercial 4.0 International), as described at http://creativecommons.org/licenses/by-nc/4.0/. 


\section{Where we've come}

Tremendous advances have been made in our knowledge of mRNA turnover over the last 20 years. Although the general pathways of mRNA decay remain constant, many of the major nucleases and axillary factors have been identified. Multiple deadenylases have been uncovered, PARN, Pan2/Pan3, and the CCR4/Not complex, all functioning to remove the $3^{\prime}$ terminal poly (A) tail. The deadenylated mRNA can be degraded by the exosome complex consisting of a 9-protein core arranged in a ring structure with its $3^{\prime}$ catalytic exonuclease activity provided by either an associated PM/Scl-100 (Rrp6 in yeast) or Rrp44 (Dis3 in yeast) proteins with Rrp44/Dis3 also providing an endonuclease activity. Following decay of the RNA body from the $3^{\prime}$ end, the remaining cap structure is hydrolyzed by the scavenger decapping enzyme DcpS (Dcs1 in yeast). For mRNAs undergoing $5^{\prime}$ end decay, at least two Nudix hydrolase proteins, Dcp2 and Nudt16, have been identified that directly decap the mRNA. Following $5^{\prime}$ end decapping Xrn1 still remains the best characterized nuclease that degrades uncapped $5^{\prime}$-end monophosphorylated RNAs, although the recently identified DXO family dual nucleases that can both decap a normal or incompletely capped $5^{\prime}$ end and subsequently degrade the resulting $5^{\prime}$ monophosphorylated RNA by an intrinsic $5^{\prime}$ to $3^{\prime}$ exonuclease activity suggests there are more. A novel twist to the mechanism of mRNA decay has been the realization that deadenylated mRNAs can undergo an oligouridylation intermediate at the $3^{\prime}$ end that promotes decay of the mRNA from either end. The prevalence of N6-methyladenosine within mRNAs and its correlation to mRNA turnover was also unexpected. Moreover, the potential for cytoplasmic recapping has raised new prospects of pathways that may recycle decapped mRNAs for reintegration into the translational pool.

Considerable progress has also been made in the modulation of mRNA decay. This process involves an extensive list of proteins that recruit the deadenylases, exosome or decapping enzymes as well as accessory proteins that stimulate the respective nuclease activities. The significance of modulating mRNA levels has further been strengthened by the discovery of multiple levels of quality control incorporated into RNA metabolism. These controls include a surveillance mechanism to detect and degrade mRNAs that are incompletely capped, mRNAs containing paused ribosomes (no-go decay) and mRNAs that lack a translation stop codon (nonstop decay). NMD remains the best-characterized surveillance mechanism at the molecular level. In addition to uncovering a role for the SMG6 endonuclease in NMD, extensive sensing mechanisms have been identified to detect mRNAs harboring a premature termination codon (PTC). These include the deposition of exon-junction complex proteins to demark splice sites and enable detection of the relative positioning of a putative PTC or the abnormally long distance of a PTC from the polyA tail as parameters distinguishing normal from premature stop codons. A significant new understanding in the past decade has been the realization that NMD factors are also involved in general and specialized decay pathways distinct from NMD including a central role for UPF1 in Staufen-mediated mRNA decay or in the decay of the nonpolyadenylated histone mRNAs.

Beyond advances in the identification of cis elements and trans factors involved in mRNA turnover, an emerging theme has been the coordination between mRNA synthesis and decay. A buffering system has been uncovered in yeast whereby the degradation of an mRNA and reduction of its steady state levels are compensated by transcriptional upregulation. The Xrn1 exonuclease appears to be the critical factor that mediates the interplay. Similarly, compartmentalization of mRNA decay factors into distinct cytoplasmic foci termed Pbodies or stress granules and the inverse correlation between translationally competent mRNAs in polysomes or silenced mRNAs in the foci provide further potential for coordinated regulation.

\section{Where we might be going}

Very few could have imagined the advances in RNA biology we have been witnessing, and it seems we are only at the beginning of the RNA revolution. It is difficult to envision what the next 20 years entail. A great wealth of knowledge has been amassed in deciphering the molecular mechanism involving mRNA decay. Undoubtedly there is still much more that will be uncovered regarding the basic molecular mechanisms underpinning regulated mRNA decay including new nucleases, RNA binding protein modulators and RNA elements/structures and the signaling pathways that control them. The field is poised for a more systems-wide integration of the individual regulatory mechanisms into a coordinated network of controls at the cellular, tissue and organismal levels. Uncovering the circuitry of modulation influenced by each decay factor in both normal and disease states will provide a holistic understanding of regulated mRNA turnover and its global function. What are the physiological consequences of the decay factors and the targets they modulate? How does the decay machinery synergize with other cellular processes? Beyond mRNA decay, does turnover of noncoding RNAs mimic mRNA decay? How are their stabilities regulated and what physiological role does the modulation of their half-lives fulfill? How can the different nucleases, pathways and/or specific transcript half-lives be manipulated for therapeutic outcomes? We are already seeing RNA based therapeutics with positive efficacies in clinical trials at several levels including nonsense-codon suppression and splice switching technologies. It's feasible that future approaches to target transcript-specific, or class-specific, modulators of mRNA turnover will be implemented in strategies to ameliorate, if not cure, disease. 


\section{RNA}

The overall impact of the RNA journal in advancing the broader RNA field and promoting the significance of post-transcriptional control cannot be overstated. The mRNA turnover field is no exception, with many outstanding papers directly addressing questions in mRNA decay in- cluded within the more than 400 papers published on this topic within its pages. Lastly, the success of the journal from its infancy in 1995 to its current prominence may not have been possible without the dedication of its Editor in Chief from the onset, Tim Nilsen. Happy 20th Anniversary RNA and a great big Thank You from all of us to Tim! 

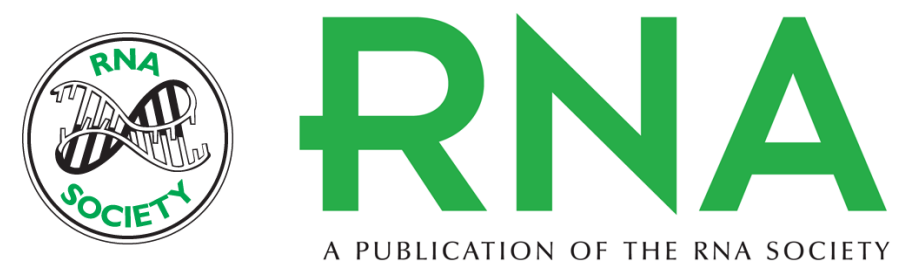

A PUBLICATION OF THE RNA SOCIETY

\title{
Twenty years of RNA and mRNA decay
}

\author{
Megerditch Kiledjian
}

RNA 2015 21: 664-666
Open Access Freely available online through the RNA Open Access option.
Creative This article, published in $R N A$, is available under a Creative Commons License Commons (Attribution-NonCommercial 4.0 International), as described at License http://creativecommons.org/licenses/by-nc/4.0/.
Email Alerting Receive free email alerts when new articles cite this article - sign up in the box at the Service top right corner of the article or click here.

\section{IIII!II Providing Precise Solutions for your research.}

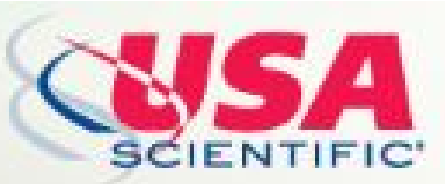

To subscribe to $R N A$ go to:

http://rnajournal.cshlp.org/subscriptions

(C) 2015 Kiledjian; Published by Cold Spring Harbor Laboratory Press for the RNA Society 